\title{
Total hip replacement: indications for surgery and risk factors for failure
}

Total hip replacement is one of the most successful and cost effective interventions in medicine. ${ }^{12}$ It offers reliable relief of pain and considerable improvement in function in patients suffering with osteoarthritis or inflammatory arthritis of the hip..$^{3-7}$ Currently about 50000 hip replacements are performed in the United Kingdom annually while worldwide the number is over 300000 . Two thirds of these are performed in patients over 65 years of age. ${ }^{8}$ Ninety to ninety five per cent of patients can expect to have their total hip replacement functioning at 10 years, ${ }^{8}$ and in $85 \%$ they will still be functioning at 20 years. ${ }^{9}$

Although $85 \%$ of patients undergoing total hip replacement have a diagnosis of osteoarthritis, ${ }^{8}$ inflammatory arthritis, both seropositive and seronegative, is an important indication for total hip replacement and can offer tremendous improvements in quality of life even in the very young. ${ }^{10}$ In rheumatoid arthritis it has been estimated that the cost to the community of total hip replacement will be recuperated within an average 1.5 years because of savings made on medical and social care. ${ }^{2}$

Quality of life after surgery approximates that of a healthy reference population. ${ }^{4}$ Improvements in pain, energy levels, sleep, social, and sexual function are all observed. ${ }^{4}$ Oxygen demands on activity are decreased and walking ability improves. ${ }^{511}$ Most of these improvements are seen within three months of surgery. ${ }^{5}$ These gains in quality of life allow large numbers of patients to retain their independence and function more actively in society.

\section{Indications for surgery}

PAIN

Pain is the principal indication for hip replacement and is reliably relieved as early as one week after surgery. ${ }^{12}$ Pain from an arthritic hip is classically located in the groin and buttock. Radiation into the thigh may occur and at times pain may present in, or even below the knee. The pain is usually described as a dull ache that is difficult to localise. Activity aggravates the pain while rest relieves it. Increased activity during the day may be followed by pain extending into the evening. Night pain is particularly distressing to patients and an important surgical indication.

FUNCTIONAL LIMITATIONS

In osteoarthritis functional limitations are usually associated with pain but are rarely an indication for hip replacement in isolation. Walking and particularly stair climbing become difficult, with start up pain a particular feature. Capsular contractions and joint deformity cause a decreased range of motion in the hip, which typically leads to patients complaining of problems with pedicure and putting on shoes and socks or stockings. Functional limitations are usually more severe in patients with inflammatory arthritis reflecting the systemic nature of their disease. Functional improvements can be confidently predicted in patients with osteoarthritis and rheumatoid arthritis after total hip replacement.

STIFFNESS

There are certain patient subgroups in which joint stiffness without hip pain is an indication for surgery. In ankylosing spondylitis hip stiffness, or even ankylosis, can leave patients with tremendous functional disability in the absence of pain. Hips commonly fuse in flexion, which in combination with a stiff thoracolumbar spine contributes to a stooped posture. These patients may become bed bound and if mobile often require walking aids. Hip replacement in this group leads to dramatic improvement in function allowing bedridden patients to ambulate and most patients to discard walking aids. ${ }^{13}$

Patients who have had previous hip fusion, either spontaneous, usually from childhood infection, or surgical may also present with disability in the absence of hip pain. Disability in this group can be caused by back pain, pain in the knees or the opposite hip, as well as the disability imposed by the stiff hip. Although a hip replacement in this group of patients is technically demanding and associated with a higher complication rate than normal a moderately good range of movement can be achieved. Furthermore some surgeons feel that an arthrodesis should be taken down before another affected joint is replaced so as to minimise the risk of failure of the arthroplasty.

AGE

While most hip replacements are performed in patients between 60 and 80 years of age, older or younger age is not a contraindication to surgery. Hip replacement is occasionally performed in patients in their teens and early twenties. In this age group its most successful indication has been in relieving pain and improving function in patients with debilitating childhood inflammatory arthritis. In one study of patients 21 years or younger over $90 \%$ implant survival was seen at 10 years in patients with juvenile arthritis. ${ }^{14}$ This severely affected group of patients enjoyed a greatly improved quality of life over this period. Twenty five per cent of North American surgeons in one survey considered a patient's age over 80 as a factor against total hip arthroplasty. ${ }^{15}$ We do not agree that older age should preclude a patient from total hip replacement and we do not think there is an upper chronological age beyond which patients should not be considered for total hip replacement. Elderly patients undergoing elective hip replacement for either osteoarthritis or rheumatoid arthritis have a greater life expectancy than average ${ }^{16}$ probably reflecting less co-morbidity in patients considered fit for elective surgery.

RADIOGRAPHIC CHANGES

The severity of the radiographic changes of arthritis within the hip joint usually but not always reflects the severity of the patients' disability. However the decision to perform surgery is made on the severity of the symptoms not the severity of the radiographic changes. At times, particularly in osteoarthritis associated with a more pronounced inflammatory response, radiographic changes can be mild in association with severe symptoms. It is important to appreciate that mild radiographic signs of disease should not exclude a patient from consideration for total hip replacement. Occasionally, in the presence of mild radiographic changes, it is not possible to be certain that the hip is the source of pain. In this situation we find that an injection of local anaesthetic into the hip, performed under radiographic control, is a very useful diagnostic test.

RELATIVE CONTRAINDICATIONS

Factors considered as poor prognostic indications for total hip replacement are significant medical disease where the risk of surgery outweighs the expected benefit, psychiatric disease, dementia, or systemic infections. Though obesity 
and in particular morbid obesity are considered relative contraindications to hip replacement studies have shown that these patient groups can undergo hip replacement with low complication rates, with significant improvements in functional level, and at least in the short-term no increased risk of implant loosening. ${ }^{17}$ Local considerations against hip replacement include poor vascular supply, poor soft tissue cover, ulcers, and neuropathic disease of the hip.

\section{SURGICAL ALTERNATIVES}

In younger patients with non-inflammatory arthritis consideration should be given to alternative types of surgical intervention such as joint preserving procedures or possibly arthrodesis. Joint preserving procedures such as acetabular augmentation, femoral or acetabular osteotomy are usually considered if there are mechanical abnormalities within the hip such as acetabular dysplasia that predisposes to osteoarthritis. The aim is to improve the biomechanics of the hip, to improve the patient's symptoms, and to delay the need for joint replacement surgery. ${ }^{18}{ }^{19}$ These procedures have high success rates provided the selection criteria are appropriate, and can be converted to total hip arthroplasty at a later date if indicated. Hip arthrodesis is most ideally utilised in the young active otherwise healthy patient with unilateral hip disease and a normal lumbar spine. When correctly indicated reliable relief of pain is achieved with patients complaining of little disability provided the hip is fused in a functional position. Low back pain, ipsilateral knee pain, and degenerative arthritis in the contralateral hip can limit the life span of an arthrodesis however these are usually relieved by conversion of the arthrodesis to a total hip replacement. Successfully functioning arthrodeses in $79 \%$ of patients at an average 35 year follow up illustrates the durability of this procedure. ${ }^{20}$

\section{Reasons for failure}

Failure of a hip replacement requiring revision surgery occurs at a rate of approximately $1 \%$ per year for the first 15 years. Beyond eight years acetabular component loosening is more common than failure of the femoral component. Late aseptic loosening of components is the commonest reason for implant failure causing approximately $75 \%$ of failures. The three next most common reasons for implants requiring revision are infection, technical errors at the time of surgery, and recurrent dislocation. ${ }^{8}$ If loosening is seen within the first two years after surgery infection should be suspected.

Although there is some overlap it is easier to appreciate risk factors for implant failure if we consider them under patient factors, implant factors, and surgeon factors.

\section{PATIENT FACTORS}

Age, sex, and aetiology of arthritis are the most important patient factors with a bearing on implant failure caused by aseptic loosening. The Swedish hip registry, ${ }^{8}$ which records national outcomes of hip replacements, has shown a significant increase in failure rates in men compared with women with the difference apparent as early as two years after surgery. This survey also showed that regardless of the underlying diagnosis the younger the patient the higher the rate of implant failure. Fractured neck of femur and avascular necrosis of the femoral head are the diagnosis that have the reputation for the worst outcome after total hip replacement. $^{821}$

Male patients under 50 years of age, particularly with osteoarthritis secondary to trauma or avascular necrosis of the femoral head have increased rates of aseptic loosening after total hip replacement. These patients often return to athletic or heavy manual activities and this is correlated with increased implant failure at 5-10 years. Despite this implant survivorship at an average 18 year follow up in men less than 50 years of age is $80-85 \%$. $^{22}$

Female patients less than 55 years of age with rheumatoid arthritis are another higher risk group for aseptic loosening with $25 \%$ failure rates by 15 years. The changed biological activity of the host bon $\mathrm{e}^{23}$ and increased bleeding at the time of surgery are probably contributing factors to implant loosening though the aetiology of increased loosening in rheumatoid arthritis is still not clear.

Deep infection occurs in around $0.5-2 \%$ of total hip replacements and is the cause for revision surgery in at least $7.5 \%$ of failures. Infection is more common in inflammatory arthritis, psoriatic arthritis, patients taking corticosteroid treatment, chronic renal failure, diabetes mellitus, high risk surgical patients, malnutrition, and older age. ${ }^{24}$

Dislocation rates of 1-2\% can be expected in the best of hands but are often greater than $5 \%$. Most of these will be single dislocations occurring in the early postoperative period, which are successfully treated by closed reduction. One in 30 revision arthroplasties are performed as a consequence of recurrent dislocation. Dislocation rates are increased in patients over 80 years of age. Poor muscular tone, femoral neck fractures, acetabular dysplasia, cerebral palsy, muscular dystrophy, and intellectual impairment are all associated with an increased dislocation rate. ${ }^{25}$

IMPLANT FACTORS

Probably the most debated topic in joint replacement surgery is that of whether implants should be inserted with or without cement. Non-cemented implants were introduced in response to loosening seen in cemented systems. However to date the long term survival of cemented implants remains superior to any non-cemented system. A comparison of countries where hip registries are maintained shows that the higher the percentage of non-cemented implants inserted the higher the implant failure rate. ${ }^{8}$

Improved operative techniques and implant materials mean that survival of some earlier designs of hip arthroplasties continues to increase. ${ }^{8}$ Improvements in surgical and in particular cementing techniques have led to decreased failure rates of hip replacements. Unfortunately many new implants, which are often used in the absence of clinical review, are failing to match the survival rates of earlier designs. ${ }^{8}$ Furthermore virtually all implants, instrumentation, and surgical techniques are regularly modified. Although these modifications are introduced to improve results they often achieve the opposite. For example minor changes in surface finish or stem geometry have been found to have detrimental consequences. ${ }^{8}$ There are now over 60 different primary total hip prostheses available in the United Kingdom, with a tremendous variation in cost. Over half of these have been introduced in the past five years, and the vast majority have no published clinical results. ${ }^{26}$ This is clearly unsatisfactory as some or perhaps many may have poor results. Outside research centres, which must be carefully monitoring clinical data on newer implants we believe that surgeons should be inserting prostheses with published peer reviewed follow up of at least 10 years.

SURGEON FACTORS

Surgical technique is extremely important in determining implant survival. Experienced hip surgeons generally agree that it is more important to choose your surgeon than the implant. Surgical approach, preparation of the implant bed, and cementing techniques all reflect on implant 
survival. ${ }^{8}$ In a study of surgeons in Florida it was found that patients treated by surgeons doing low numbers of arthroplasties had higher death rates and that costs and length of hospital stay were greater. ${ }^{27}$ Dislocation rates do not seem to differ with surgical approach but are higher when the surgery is performed by inexperienced surgeons. ${ }^{28}$ Surgeons need to be trained to perform total hip replacement and must be carefully supervised during their learning period. Unfortunately training is difficult as the operation is complex and their is little evidence as to which are the best techniques to use. Though hip replacement is a commonly performed procedure it is not an easy operation to perform well and the implications to the patient are enormous if it is performed badly.

To determine where total hip replacement is performed based on initial cost alone may well be a false economy. Doctors should be encouraged to refer their patients for total hip replacement to surgeons who have an interest in hip surgery. These surgeons should be aware of how their failure rates compare with other institutions, as is done in hip registries in Sweden, Norway, and Finland. This should lead to a progressive improvement in results.

\section{Conclusion}

Hip replacement has transformed the lives of hundreds of thousands of people with arthritis of the hip regardless of the underlying aetiology. Surgeons should select their patients for surgery carefully, use implants with long term follow up, and apply surgical techniques that have been shown to decrease implant failure rates.

\section{Nuffield Orthopaedic Centre, Windmill Road, Headington,}

WRAWFORD Oxford OX3 $7 L D$

1 Liang M, Cullen K, Larson M, Thompson M, Schwartz J, Fossel A. Cost effectiveness of total joint arthroplasty in osteoarthritis. Arthritis Rheum 1986;29:937-43.

2 Jonsson B, Larsson S. Functional improvements and costs of hip and knee arthroplasty

3 Rissanen P, Aro S, Slatis P, Sintonen H, Paavolainen P. Health and quality of life before and after hip or knee arthroplasty. J Arthroplasty 1995;10:169-75.
4 Wiklunch I, Romanus B. A comparison of quality of life before and after arthroplasty in Paitents who had arthrosis of the hip joint. J Bone Joint Surg 1991;73A:765-9.

5 Laupacis A, Bourne R, Rorrabeck C, Feeny D, Wong C, Tugwell P, et al. The effect of elective total hip replacement on health related quality of life. J Bone Joint Surg 1993;75A:1619-26.

6 Ritter M, Albohm M, Keating M, Faris P, Meding J. Comparative outcomes of total joint arthroplasty. J Arthroplasty 1995;10:737-41.

7 McGuigan F, Hozack W, Moriarty L, Eng K, Rotham R. Predicting qualityof-life outcomes following total joint arthroplasty. J Arthroplasty 1995;10:742-7.

8 Malchau H, Herberts P. Prognosis of total hip replacement. The Swedish National Hip Arthroplasty register 1996. Proceedings American Academy of Orthopedic Surgeons. Atlanta: 1996.

9 Schulte K, Callaghan J, Kelley S, Johnston R. The outcome of Charnley total hip arthroplasty with cement after a minimum twenty year follow up. J Bone Joint Surg 1993;75A:961-75.

10 Scott R, Sarokhan A, Dalziel R. Total hip and total knee arthroplasty in juvenile rheumatoid arthritis. Clin Orthop 1984;182:90-8

11 Mattsson E. Energy cost of level walking. Scand J Rehabil Med 1989;23S:148.

12 Aarons H, Hall G, Hughes S, Salmon P. Short-term recovery from hip and knee arthroplasty. J Bone Joint Surg 1996;78B:555-8.

13 Shih L, Chen T, Lo W, Yang D. Total hip arthroplasty in patients with ankylosing spondylitis: longterm followup. J Rheumatol 1995;22:1704-9.

14 Wedge J, Cummishmey D. Primary arthroplasty of the hip in patients who are less than 21 years old. J Bone Joint Surg 1994;76A:1732-41.

15 Mancuso C, Ranawat C, Esdaile J, Johanson N, Charlson M. Indications for total hip and total knee arthroplasties. J Arthroplasty 1996;11:34-46.

16 Holmbery S. Life expectancy after THA. J Arthroplasty 1992;7:183-6.

17 Lehman D, Capello W, Feinberg J. Total hip arthroplasty without cement in obese patients. J Bone Joint Surg 1994;76A:854-62.

18 Mills M, Murphy S, Poss R. Osteotomies about the hip for the prevention and treatment of osteoarthritis. J Bone Joint Surg 1995;77A:626-47.

19 Iwase T, Hasegawa Y, Kawamoto K, Iwasada S, Yamada K, Iwata H. Twenty years' follow up of intertrochanteric osteotomy for treatment of the dysplastic hip. Clin Orthop 1996;331:245-55.

20 Callaghan J, Brand R, Pedersen D. Hip arthrodesis. J Bone Joint Surg 1985; 67A:1328-35.

21 Cabanela M. Bipolar versus total hip arthroplasty for avascular necrosis of the femoral head. Clin Orthop 1990;261:59-62.

22 Sullivan P, MacKenzie J, Callaghan J, Johnston R. Total hip arthroplasty with cement in patients who are less than 50 years old. J Bone Joint Surg 1994;76A:863-72.

23 Havdrup A, Hulth A, Telhag H. The subchondral bone in osteoarthritis and rheumatoid arthritis of the knee. Acta Orthop Scand 1976;47:345-50.

24 Hanssen A, Osmon D, Nelson C. Prevention of deep periprosthetic joint infection. J Bone Joint Surg 1996;78A:458-71.

25 Fackler C, Poss R. Dislocation in total hip arthroplasty. Clin Orthop 1980; 151:169-78.

26 Murray D, Carr A, Bulstrode C. Which primary total hip replacement. J Bone Joint Surg 1996;77B:520-7.

27 Lavernia C, Guzman J. Relationship of surgical volume to short term mortality, morbidity and hospital changes in arthroplasty. J Arthroplasty 1995; 10:133-40.

28 Hedlundh U, Ashnfelt L, Hybbinette C, Weckstrom J, Fredin H. Surgical experience related to dislocations after total hip arthroplasty. J Bone Joint Surg 1997;78B:206-9. 